\title{
HIGH VELOCITY NEUTRAL HYDROGEN CLOUDS
}

\author{
R. D. DAVIES \\ University of Manchester, Nuffield Radio Astronomy Laboratories, Jodrell Bank, \\ Cheshire, United Kingdom
}

\begin{abstract}
A review is given of the observations of neutral hydrogen high velocity clouds $\left(|v|>80 \mathrm{~km} \mathrm{~s}^{-1}\right)$ in and near the Galaxy. The positive and negative clouds are seen to have different distributions in the sky, following roughly the velocity pattern of galactic rotation. A characteristic of the majority of the clouds is their distribution in elongated bands or strings. The various theories of origin of HVCs are discussed; the possible role of the tidal interactions between the Magellanic Clouds and the Galaxy is emphasized. Tests are suggested to distinguish between the Oort theory of the infall of intergalactic material and theories which envisage the HVCs as originating in the outermost spiral structure.
\end{abstract}

\section{Introduction}

The major part of the neutral hydrogen in the Galaxy is confined to a disk-shaped region with a thickness at half intensity of a few hundred parsecs. Outside the Sun's distance $(R \gtrsim 10 \mathrm{kpc})$ a significant departure from a plane occurs in the neutral hydrogen distribution in the sense that in the sector $l \sim 20^{\circ}$ to $140^{\circ}$ (galactocentric longitudes $L=30^{\circ}$ to $160^{\circ}$ ) it lies above the galactic plane and in the sector $l=240^{\circ}$ to $340^{\circ}$ $\left(L=210^{\circ}\right.$ to $\left.330^{\circ}\right)$ it lies below the plane. (See, for example, Kerr and Westerhout, 1965.) The centres of the arms at $R \simeq 15 \mathrm{kpc}$ lie at $\sim 0.5 \mathrm{kpc}$ from the plane in these regions. One has to look outside the Galaxy for the origin of this warp in the neutral hydrogen distribution. The cause is probably the tidal effect of the Magellanic Clouds (see, for example, Hunter and Toomre, 1969).

Within the Galaxy there is evidence for departures from non-circular motions of a few tens of $\mathrm{km} \mathrm{s}^{-1}$. Rickard (1968) has reported a systematic deviation of 20 to $30 \mathrm{~km}$ $\mathrm{s}^{-1}$ in a region $30^{\circ}$ in extent on the galactic plane in Cassiopeia-Perseus. A number of investigators (Berkhuijsen et al., 1971; Fejes and Verschuur, 1973) have also found velocities of $20-30 \mathrm{~km} \mathrm{~s}^{-1}$ associated with the continuum spurs.

Although the full width at half intensity of spiral arms near the Sun is in the range 200-300 pc there are significant amounts of material (a few per cent of the peak intensity) at the velocity of the arms, and therefore belonging to them, which reach $1-2 \mathrm{kpc}$ from the galactic plane. This gas is seen for example in the Sagittarius and Perseus arms in the low latitude survey by Weaver and Williams (1973).

The present review will discuss the neutral hydrogen data which are not so obviously related to the narrow disk component. This gas is called the intermediate and high velocity gas. Much of it is in localized regions which have become known as intermediate velocity clouds (IVCs) and high velocity clouds (HVCs). Some of the gas is also in extended regions of lower brightness underlying the IVCs and HVCs. The division of clouds between IVCs and HVCs has somewhat arbitrarily been made at $|v|=70 \mathrm{~km} \mathrm{~s}^{-1}$ (Blaauw and Tolbert, 1966) or $80 \mathrm{~km} \mathrm{~s}^{-1}$ (Hulsbosch, 1973). Of course gas at velocities up to $\sim 130 \mathrm{~km} \mathrm{~s}^{-1}$ relative to the LSR is well known in the plane of 
the Galaxy and is compatible with circular motion. Indeed most IVCs are believed to be closely related to the spiral structure of the Galaxy (Blaauw and Tolbert, 1966). Our discussion will therefore be concerned with the HVCs, and it will be limited to gas with $|v|>80 \mathrm{~km} \mathrm{~s}^{-1}$.

\section{Surveys of HVCs}

The Dutch groups first made surveys for HVCs with the Dwingeloo telescope (beamwidth $=0.6^{\circ}$ ) and catalogued many HVCs (Muller et al., 1966; Hulsbosch and Raimond, 1966; Tolbert, 1971; Hulsbosch, 1968; van Kuilenberg, 1972a, 1972b). Another important survey was made at Ohio State University by Mathewson et al. (1966) and by Meng and Kraus (1970). This demonstrated that many of the brighter HVCs and IVCs are embedded in large elongated areas of low intensity emission at similar velocities. A series of high sensitivity observations of areas of the northern sky was made at a $2^{\circ}$ angular resolution with the Bell Telephone Laboratories 20 -ft horn reflector (Wannier and Wrixon, 1972; Wannier et al., 1972; Encrenaz et al., 1971). These showed extensive low intensity emission at both negative and positive velocities.

The available data on HVCs are summarized in Figure 1. This also includes the recent positive velocity data for extended emission features in the southern sky observed by Mathewson et al. (1973). They found relatively few negative velocity clouds. The dimensions plotted for the various clouds give an indication of their half intensity contours. The continuous bands of emission in which some of these concentrations lie are not shown. HVCs are plotted for $|v|>80 \mathrm{~km} \mathrm{~s}^{-1}$ with the addition of some positive velocity clouds in the range $v=+60$ to $+80 \mathrm{~km} \mathrm{~s}^{-1}$ listed by van Kuilenberg. A description of interesting features of the distribution will now be given.

(a) THE 'BAND' EXTENDING FROM $l=50^{\circ}, b=10^{\circ}$, TO $l=130^{\circ}, b=+55^{\circ}$

Figure 1 shows a quasi-continuous band of HVCs beginning at $l=50^{\circ}, b=+10^{\circ}$, passing through $l=90^{\circ}, b=+40^{\circ}$, and continuing to $l=130^{\circ}, b=+55^{\circ}$. HVCs with velocities in the range -80 to $-165 \mathrm{~km} \mathrm{~s}^{-1}$ are seen; $80 \%$ are in the range -100 to $-150 \mathrm{~km} \mathrm{~s}^{-1}$. Further, there appears to be no systematic trend of velocity along the 'band'; the mean velocity at the high latitude end is $-132 \mathrm{~km} \mathrm{~s}^{-1}$ compared with $-114 \mathrm{~km} \mathrm{~s}^{-1}$ at the low latitude end.

The data available to the present are inconclusive on the question of whether this 'band' is a physical entity. They do, however, indicate that large areas of emission are continuous in the sky. Meng and Kraus describe the area from $l=50^{\circ}$ to $80^{\circ}$ as a 'large quasi-continuous group'. Similarly, the section from $l=90^{\circ}$ to $138^{\circ}$ has a common envelope of detectable emission as shown for example in Figure $6 \mathrm{~b}$ by van Kuilenberg (1972a). This latter region includes the brighter emission which was originally designated complexes $\mathrm{C}_{\mathrm{I}}, \mathrm{C}_{\mathrm{II}}$ and $\mathrm{C}_{\mathrm{III}}$. The complexes $\mathrm{MI}_{\mathrm{I}}$ and $\mathrm{M}$ II lie at the upper latitude end of this 'band'; observations so far do not suggest a connection between $\mathrm{MI}, \mathrm{M}$ II and the 'band'.

The only break discernible in the published data for the 'band' is in the vicinity of $l=80^{\circ}, b=+30^{\circ}$, and even then there is an isolated cloud at $l=77^{\circ}, b=+31^{\circ}$ (Ohio 


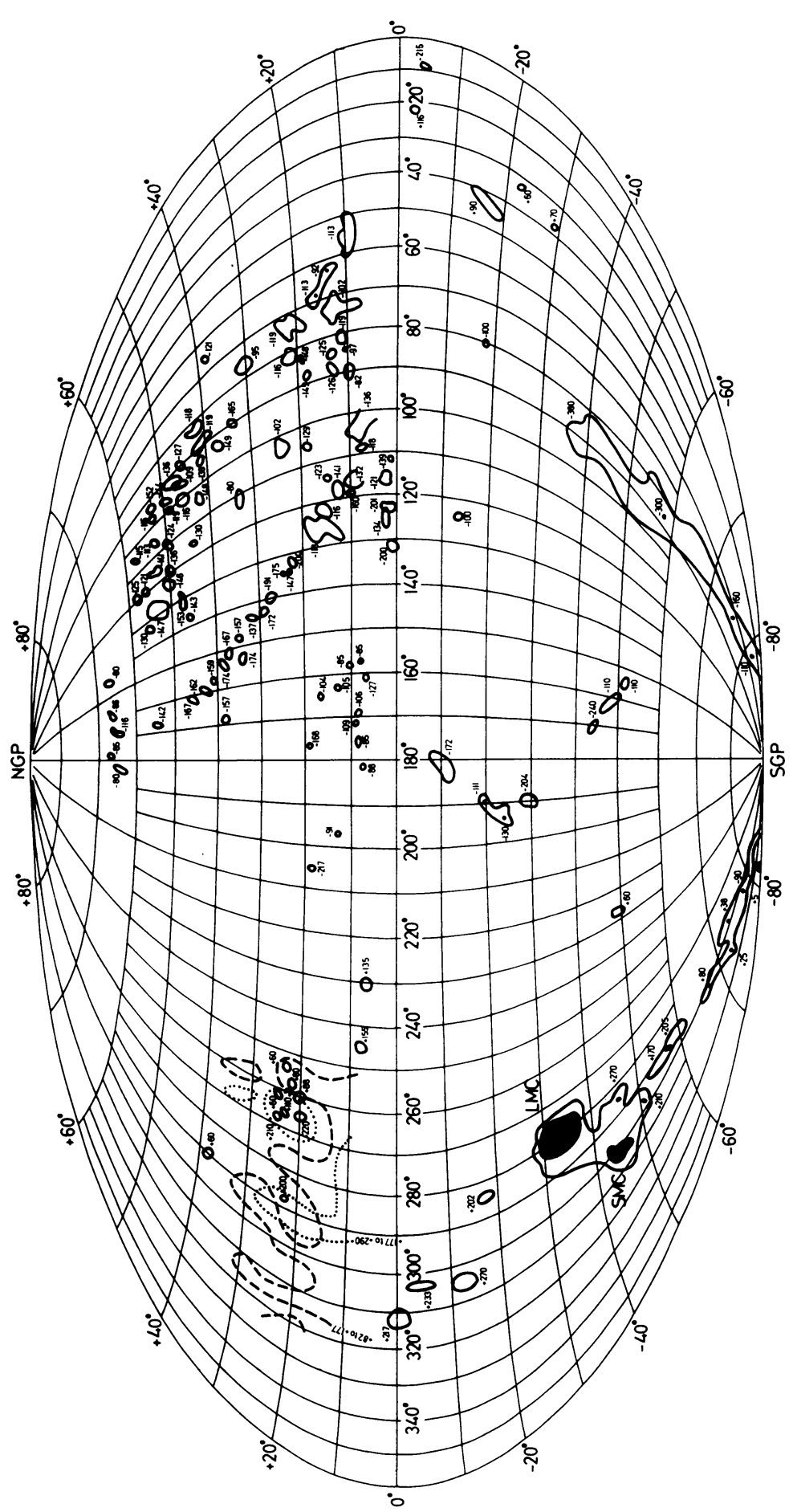

를

के ซึ

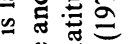

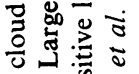

氖点吕

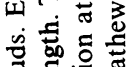

은 可

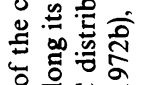

ก

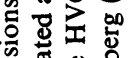

氖苛

E.

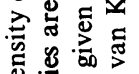

氖的

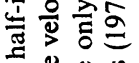

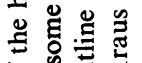

¿

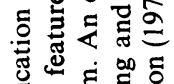

๑ึّ

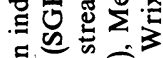

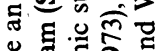

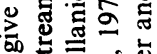

की

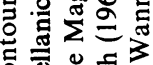

ㅇㅝㅀㅀㅀㅇ

\& $\Sigma^{\pi} 0$ :

过费焉

氜论

造券远

号志

통

焉哇苋

ప.

ن용

证

뎐

놀

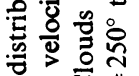

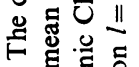

-

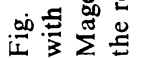


cloud OTH 468). High sensitivity observations will be required to detect any emission link through the region of the break.

Another interesting feature of the lower latitude section of this 'band' is the clear indication of a fork at $l=65^{\circ}, b=+12^{\circ}$. A lower arm of the fork runs at constant galactic latitude to $l=90^{\circ}, b=+12^{\circ}$. It has similar velocities to those seen in the upper arm.

(b) THE BAND EXTENDING FROM $l=100^{\circ}, b=+7^{\circ}$, TO $l=168^{\circ}, b=+54^{\circ}$

This band, which can first be distinguished at $l=100^{\circ}, b=+7^{\circ}$, and extends to $l=168^{\circ}, b=+54^{\circ}$, is the best defined band of HVCs at northern galactic latitudes. The high latitude end of this band was originally designated complex $A$. More sensitive studies (Hulsbosch, 1968; Giovanelli et al., 1973) have shown that it is a quasicontinuous feature which extends for $30^{\circ}$, and its width is seldom more than $3^{\circ}$. It consists of bright small diameter features superposed on a low brightness background; these small-scale features will be discussed in Section V. Velocity differences in adjacent complexes may be as much as $50 \mathrm{~km} \mathrm{~s}^{-1}$.

The low latitude end of this band merges into the galactic plane neutral hydrogen near $l=100^{\circ}, b=+7^{\circ}$, which has velocities at least as negative as $-130 \mathrm{~km} \mathrm{~s}^{-1}$. This situation is illustrated in Figure 6 of Hulsbosch (1973), where the band can be seen superposed on extensive galactic plane emission. High sensitivity observations in sections across the galactic plane at $l=100^{\circ}$ and $120^{\circ}$ are shown in Figure 2 . These show the extended nature of the emission at $v<-96 \mathrm{~km} \mathrm{~s}^{-1}$. The peaks on the
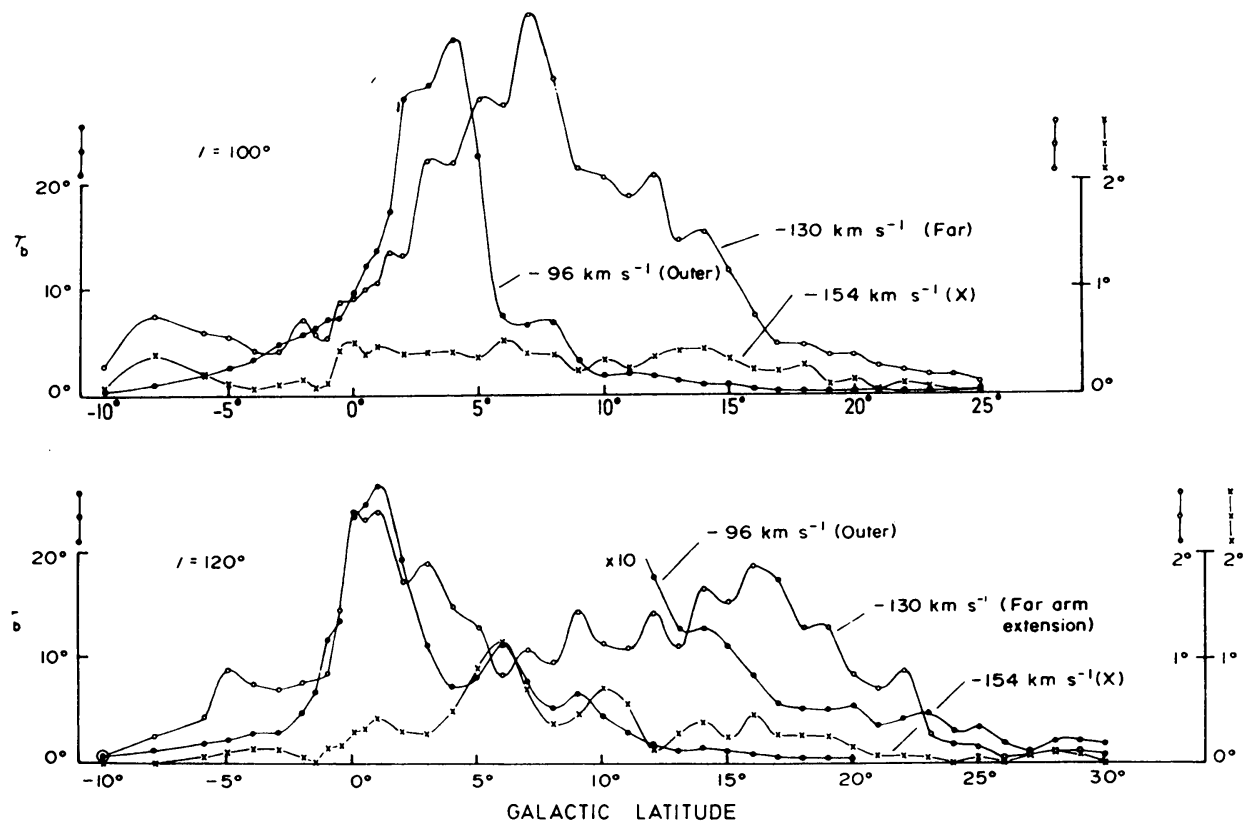

Fig. 2. Sections across the galactic plane at $l=100^{\circ}$ and $120^{\circ}$ showing the broad distribution of high velocity gas at velocities in the range -96 to $-154 \mathrm{~km} \mathrm{~s}^{-1}$ (from Davies. 1972). 
$v=-130 \mathrm{~km} \mathrm{~s}^{-1}$ plots in both sections were recognized as HVCs in early work and are plotted as individual clouds in Figure 1.

The existence of weak emission between the high latitude end of the band discussed in this section and the band discussed in Section IIa has been detected by Encrenaz et al. (1971). It is not clear whether it is physically associated with either of the bands; it could even be a connecting bridge between the two bands.

(c) THE BANDS OF EMISSION IN THE ANTICENTRE REGION

A number of bands of emission having substantial negative velocities are seen in the anticentre region. These are of particular interest since they cannot be understood in terms of any circular model of galactic rotation.

One of these bands runs approximately parallel to the galactic plane at $b=+8^{\circ}$ between $l=152^{\circ}$ to $192^{\circ}$. The principal emission peaks lie in the velocity range -70 to $-130 \mathrm{~km} \mathrm{~s}^{-1}$. This was labelled feature $Q$ by Kepner (1970), arm $U$ by Davies (1972) and arm $E$ by Verschuur (1973).

Other elongated features are found along the line $l=180^{\circ}, b=-10^{\circ}$, to $l=195^{\circ}$, $b=-25^{\circ}$. These have been designated the anticentre complex (AC). Another string of HVCs is found along the line $l=154^{\circ}, b=-52^{\circ}$, to $l=171^{\circ}, b=-44^{\circ}$. These two lines contain clouds with a large negative velocity range $\left(-110\right.$ to $\left.-240 \mathrm{~km} \mathrm{~s}^{-1}\right)$.

(d) POSITIVE VELOCITY CLOUDS IN THE REGION $l=250^{\circ}$ TO $320^{\circ}, b=+10^{\circ}$ TO $+30^{\circ}$

A survey of positive velocity hydrogen above the plane in the range $l=252^{\circ}$ to $322^{\circ}$ has been made by Wannier et al. (1972) with a high sensitivity system capable of detecting a neutral hydrogen column density of $\sim 4 \times 10^{18} \mathrm{~cm}^{-2}$. A wealth of positive velocity emission was seen in this area with velocities extending to $+280 \mathrm{~km} \mathrm{~s}^{-1}$. Figure 1 indicates the areas of emission in the two velocity ranges +82 to $+177 \mathrm{~km} \mathrm{~s}^{-1}$ and +177 to $+290 \mathrm{~km} \mathrm{~s}^{-1}$. The previously discovered positive velocity HVCs of Kerr (1964) and van Kuilenberg (1972a, b) lie within or adjacent to this area. The survey of Wannier et al. was taken on a $2^{\circ} \times 2^{\circ}$ grid with a $2^{\circ}$ beamwidth, and accordingly there is no information on the fine structure which can be compared with the structure of the negative velocity gas in the northern sky.

\section{(e) THE SOUTH GALACTIC POLE FEATURE AND THE MAGELLANIC STREAM}

A high velocity complex was first observed near the South Galactic Pole (SGP) by Dieter (1965) and Hulsbosch and Raimond (1966). Subsequent observations by Hulsbosch (1968) and van Kuilenberg (1972a) showed a narrower region some $30^{\circ}$ in extent extending to their southern horizon. It was unusual in having a velocity gradient along its length; more negative velocities are at the northern end. The very remarkable nature of this band was demonstrated by the high sensitivity survey of Wannier and Wrixon (1972) who found a lower intensity extension northwards to $l=90^{\circ}$, $b=-35^{\circ}$. At this point the mean velocity relative to the LSR was $-380 \mathrm{~km} \mathrm{~s}^{-1}$.

The origin of this band was elucidated by the southern sky survey of HVCs by Mathewson et al. (1973) who showed that this feature emanated from the vicinity of 
the Magellanic clouds. This filament, which they named the Magellanic Stream, follows a great circle over an arc $150^{\circ}$ long; they also describe an extension on the far side of the Magellanic Clouds which reaches to the galactic plane near $l=306^{\circ}$. The velocity of the main Magellanic stream changes monotonically from a value of -380 $\mathrm{km} \mathrm{s}^{-1}$ at $l=90^{\circ}, b=-30^{\circ}$, to $+250 \mathrm{~km} \mathrm{~s}^{-1}$ in the vicinity of the Magellanic Clouds.

\section{(f) A SUMMARY OF THE HVC PROPERTIES}

Several dominant characteristics of HVCs emerge from their distribution as plotted in Figure 1. The first is the preponderance of negative velocities in the region at positive latitudes between $l=40^{\circ}$ and $190^{\circ}$. This is within the region first surveyed at northern observatories and led to a belief that HVCs were principally negative velocity objects. The positional asymmetry about the galactic plane in this longitude range is in the sense of the well known tilt of the galactic plane; the possible significance of this fact will be discussed in later sections. The HVC velocities in the southern sky are mainly positive. Thus the sense of the velocities in both the northern and southern Galaxy are the same as produced by galactic rotation at the equator. The magnitude of the velocities, however, ranges up to values somewhat larger than normally expected for galactic rotation.

Another feature of the HVCs already commented upon in Section II and clearly indicated in Figure 1 is their confinement to elongated strings or bands. This is most vividly demonstrated in the band extending from $l=100^{\circ}, b=+7^{\circ}$, to $l=160^{\circ}, b=+$ $+46^{\circ}$. Other groups of HVCs in Figure 1 lie within elongated emission features. So far relatively little high angular resolution work has been done on the positive latitude regions between $l=250^{\circ}$ to $320^{\circ}$, and it is not yet possible to say whether much elongated structure exists there.

Probably the most remarkable feature discovered so far is the Magellanic stream. It shares with some other HVCs the elongated band structure; it contrasts with them in possessing a large velocity gradient, at least when measured relative to the LSR, although this difference would disappear if the velocities were corrected for the effects of galactic rotation. The question of whether the Magellanic stream is a completely different feature from the majority of the HVCs described above will be discussed in later sections.

On the basis of the foregoing description of the main properties of the distribution of the HVCs we will now consider theories of their origin.

\section{Extragalactic Interpretations of HVCs}

The characteristic of HVCs shown by the early observations which appeared to distinguish them from the well-known galactic spiral arm gas was their greater range in velocity and greater spread in latitude. Furthermore, the clouds seemed, at least in the low sensitivity observations then available, to be isolated features. These facts led Oort $(1966,1969,1970)$ to suggest that the HVCs were principally galactic gas swept up by the infall of intergalactic matter. Verschuur (1969) and Kerr and Sullivan (1969) 
considered the possibility that the HVCs were intergalactic clouds either at distances for a few hundreds or a few tens of kiloparsecs.

\section{(a) THE HYPOTHESIS OF OORT}

The preponderance of negative velocities found in the early observations of HVCs was interpreted by Oort $(1966,1969,1970)$ to indicate that they were gas clouds moving into the Galaxy. He supposed that intergalactic material coming in at the free-fall velocity of $\sim 500 \mathrm{~km} \mathrm{~s}^{-1}$ (as seen at the Sun) was slowed down to the observed velocities of $\sim 120 \mathrm{~km} \mathrm{~s}^{-1}$ on sweeping up about three times their mass of galactic gas. This material was supposed to be in the halo of the Galaxy or in the upper reaches of the spiral arms. The corresponding rate of inflow of intergalactic gas was $1-2 M_{\odot}$ per year, which would increase the mass of the Galaxy by $1 \%$ per $10^{9} \mathrm{yr}$. The intergalactic density in the vicinity of our Galaxy would be $\sim 3 \times 10^{-4}$ atoms $\mathrm{cm}^{-3}$. In order to maintain the galactic halo which supplies $\frac{3}{4}$ of the infalling mass in the HVCs, Oort proposed that superexplosions eject material from the galactic plane.

On this model the cloudy structure of the HVC gas is a consequence of irregularities in the halo. The problem of cooling the intergalactic gas after collision with the halo gas is overcome by the presence of coolants in the halo component which originated on the galactic plane (Savedoff et al., 1967).

The Oort model has difficulty in explaining the large body of positive velocity material in the southern sky. Also it has no immediate explanation of the elongated band-like structure of the HVC complexes making large angles with the galactic plane.

\section{(b) HVCS AS LOCAL GROUP OBJECTS}

Verschuur (1969) explored the possibility that the HVCs may be aggregates of matter (protogalaxies?) in the Local Group of galaxies which have not contracted to galactic dimensions or densities. He showed that the galactocentric velocities of HVCs and Local Group galaxies were similar. If the HVCs are required to be gravitationally stable their distances must be at least several hundred kpc. At a distance of $\sim 400 \mathrm{kpc}$ the $\mathrm{HI}_{\mathrm{I}}$ masses of the complexes are $10^{9}-10^{10} M_{\odot}$. These parameters Verschuur argued would be consistent with the HVCs being protogalaxies in the Local Group.

\section{(c) HVCs AS SATELlites OF THE GALAXY}

Kerr and Sullivan (1969) also proposed that the HVCs were extragalactic but at distances such that they may be satellites of the Galaxy. They were able to derive orbits which fitted the galactocentric velocities and sky distribution of the HVCs with $|b|=20^{\circ}$ to $75^{\circ}$. The best-fitting orbits had (i) semimajor axes in the range 30 to $80 \mathrm{kpc}$, (ii) eccentricities of 0.5 to 0.8 and (iii) inclinations to the galactic plane of $40^{\circ}$ to $70^{\circ}$. These distances are comparable with those of the Magellanic Clouds $(55 \mathrm{kpc})$. The total mass of $\mathrm{H} \mathrm{I}$ in HVCs would be $\sim 2 \times 10^{8} M_{\odot}$, a value of the same magnitude as the Magellanic Clouds. Kerr and Sullivan then comment favourably on the suggestion by Toomre that galactic tidal forces may have broken this material away from 
the Magellanic Clouds during an earlier passage. Subsequent events have shown that such an explanation is applicable to the Magellanic stream - although it may not be applicable to all the northern HVCs which show close connections with the galactic plane $\mathrm{H}$ I.

(d) SHELL OF INFALLING MATERIAL

Dieter (1971) made a high sensitivity survey of the neutral hydrogen in the northern sky within $\pm 15^{\circ}$ of the galactic plane. On the basis of these observations a model was constructed of the high velocity gas which included an infalling intergalactic component along the lines envisaged on the Oort model.

Dieter found extensive high negative velocity emission near the galactic plane which was intimately connected with the low velocity material. The lower latitude HVCs were situated in the extended emission. The maximum observed radial velocity varied with longitude but not exactly in the way expected for galactic rotation. These observations were explained in terms of a shell of high velocity gas that outlines the outermost spiral arms and extends $\pm 3.5 \mathrm{kpc}$ from the galactic plane. This gas has a rotational velocity appropriate to its distance from the centre with an additional infall velocity of $125 \mathrm{~km} \mathrm{~s}^{-1}$ directed towards the galactic centre. The mass of infalling material situated at the outer edge of the Galaxy would be $\sim 2 \times 10^{8} M_{\odot}$. Dieter follows Oort in ascribing this infall to intergalactic gas falling towards the centre and colliding with the outer regions of the Galaxy, thereby being slowed down to $125 \mathrm{~km}$ $\mathrm{s}^{-1}$. The radial motion is a remnant from galaxy formation and is taken to be principally in the galactic plane. This model does not seek to explain the HVCs at higher latitudes $\left(|b|>15^{\circ}\right)$ nor does it account for the extensive positive velocity emission in the southern sky.

\section{High Velocity Gas and Galactic Structure}

The alternative approach to the understanding of the HVCs is to consider them as an integral part of the outer galactic spiral arm system. The well-observed outer spiral structure can be traced out to velocities of $\pm 130 \mathrm{~km} \mathrm{~s}^{-1}$. Emission at these velocities, although within the range classified as high velocity clouds, is strongest on or near the galactic plane. Habing (1966) demonstrated that in the longitude range $l=42^{\circ}$ to $142^{\circ}$ this negative velocity emission extended above the plane and was still strong at $b=+10^{\circ}$ to $+15^{\circ}$. A more extensive survey of the region $l=48^{\circ}$ to $228^{\circ}, b=+6^{\circ}$ to $+20^{\circ}$, by Kepner (1970) revealed a number of high velocity features extended in longitude and latitude which were not simple continuations of known spiral arm structures. Some of these are illustrated in Figure $3 \mathrm{a}$, where velocities of up to $-150 \mathrm{~km} \mathrm{~s}^{-1}$ are seen. However, following the Oort model, she chose to explain the five features $O^{*}$, $O P^{*}, P^{*}, Q$ and $R$ as 'high velocity features resulting from collisions with intergalactic gas'. Outer extensions of the well-documented spiral arms were traced to $1-2 \mathrm{kpc}$ above the plane in many cases.

A more direct argument for the majority of the HVCs to be associated with outer 


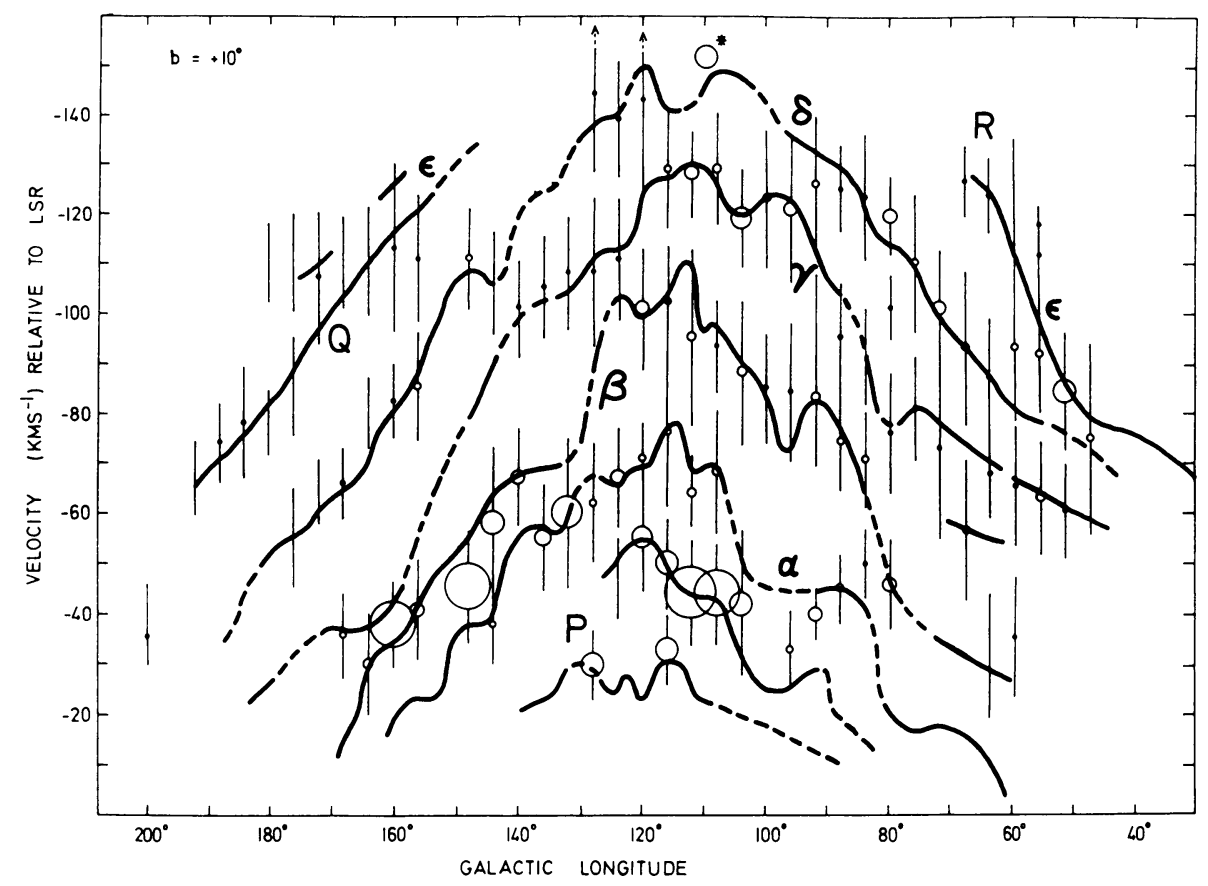

Fig. 3a. The longitude-velocity diagram for negative velocities at $b=+10^{\circ}$ according to Verschuur (arms $\alpha, \beta, \gamma, \delta$, and $\varepsilon$ shown as heavy lines) and to Kepner (as vertical lines through circles whose diameters indicate the intensity.

spiral arms was made on the basis of extensive new observations by Davies (1972, 1973) and Verschuur (1973) independently. Davies made latitude cuts at fixed longitudes around the plane which showed the intimate connection of high velocity material with the galactic plane. The latitude cuts at $l=100^{\circ}$ and $120^{\circ}$ are shown in Figure 2, which illustrate the broad distribution of negative velocity gas about the galactic plane, with a dominant extension above the plane. The brighter peaks in these cuts were previously classified as HVCs. Verschuur's observations consisted of longitude cuts at $b=+5^{\circ},+10^{\circ}$ and $+15^{\circ}$. The velocity versus longitude plots from these two series of observations are shown in Figures $3 a$ and $3 b$. They outline welldefined ridges or arm-like structures lying outside the well-known arms with velocities in the range -150 to $+150 \mathrm{~km} \mathrm{~s}^{-1}$ and can be traced in both the northern and southern skies. It was argued that these features had the characteristics of spiral arms, viz. (i) they appear as separate velocity features on the spectra, (ii) they are restricted in latitude to near the galactic plane, (iii) they are extended in longitude by at least several times their extent in latitude and (iv) they show changes in velocity with longitude consistent with galactic rotation. Wannier et al. (1972) also showed that there was extensive emission in the region $l=252^{\circ}$ to $322^{\circ}$ and $b=+10^{\circ}$ to $+30^{\circ}$ at velocities in the range +82 to $+290 \mathrm{~km} \mathrm{~s}^{-1}$ which they suggested was most likely a highlatitude extension of the outer spiral arms. 


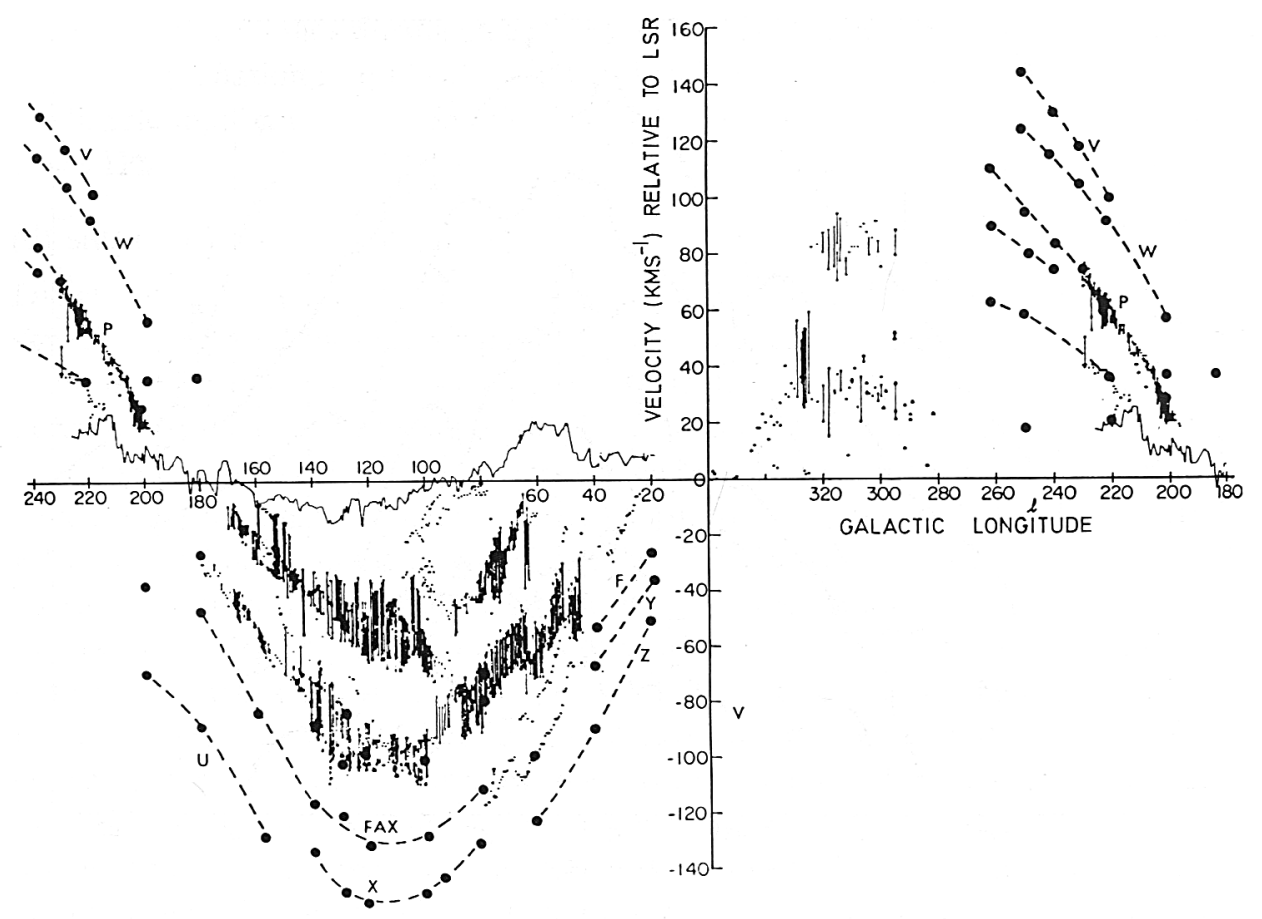

Fig. 3b. The longitude-velocity diagram for features near the galactic plane in the longitude range $20^{\circ}$ to $260^{\circ}$. This shows the high velocity 'spiral arm' features studied by Davies (1972) superposed on the bright features plotted by Weaver (1971).

There can be little doubt now that the majority of the H I at low latitudes (say $|b|<15^{\circ}$ to $20^{\circ}$ ) is intimately connected with the Galaxy and its outer spiral structure. Many of the features classified as HVCs and plotted in Figure 1 are a part of these spiral arms. A characteristic of the high velocity gas distribution in the region $l=40^{\circ}$ to $180^{\circ}$ is the preponderance of material at positive latitudes; this extends the pattern already well known as the tilt of the plane of the stronger spiral arms outside the Sun's distance. The actual location of these outer spiral arms is difficult to assess with any precision because the galactic rotation curve is not well-determined in the outer parts of the Galaxy, and moreover systematic velocities of a few tens of $\mathrm{km} \mathrm{s}^{-1}$ which are found elsewhere will produce further uncertainties in position. They are obviously outside the well-determined outer arms $(R \sim 15 \mathrm{kpc})$. Davies and Verschuur place the arms in the range $R=15 \mathrm{kpc}$ to perhaps $25 \mathrm{kpc}$ or more.

Davies argued that those $\mathrm{HVC}$ in the bands emanating from near the galactic plane (e.g., those beginning near $l=50^{\circ}, b=+8^{\circ}, l=100^{\circ}, b=+6^{\circ}$, and $l=158^{\circ}, b=+8^{\circ}$ ) were also part of the outermost spiral structure. These bands were envisaged as being warped further out of the galactic plane than the nearer spiral arms. This greater displacement from the galactic plane is of the magnitude and sense that would be expected in theories where gravitational tides are produced in the Galaxy by the Magel- 
lanic Clouds. The small fraction of HVCs with velocities grossly different from values expected for galactic rotation, for example those at high latitude and those in the anticentre at large negative velocities, were supposed to have been wrenched out of the Galaxy by the tidal forces. This is in accordance with the tidal theory and observations of external galaxies (Hunter and Toomre, 1969; Toomre and Toomre, 1972; Wright, 1972; Clutton-Brock, 1972; Eneev et al., 1973). No new phenomenon is required to explain any of the HVC data.

Verschuur's discussion of the relationship between HVCs and outer spiral arms essentially follows the discussion outlined above - emphasizing the physical connections between HVCs and spiral arms near the plane. His explanation of the noncircular motions includes the further possibilities that they may be due to a magnetohydrodynamic wave running along a spiral arm or to the winding-up of the outer spiral arms.

\section{Fine Structure in HVCs}

The early surveys of HVCs showed that both individual HVCs and the structures within high velocity complexes had sizes of a few degrees. The velocity width at half intensity points was measured to be 20 to $25 \mathrm{~km} \mathrm{~s}^{-1}$ (Hulsbosch, 1973). The structure in IVCs was found to be similar.

Observations with higher resolution in angle and velocity as well as with higher sensitivity showed that this is an oversimplified picture. Many of the HVCs reveal finer structure as may be seen in Figures $4 \mathrm{a}$ and $4 \mathrm{~b}$ taken from Davies and Buhl (1974). These diagrams are RA-velocity cuts through the HVC complexes A and MII with the $10^{\prime}$ beam of the NRAO 300-ft telescope at Green Bank. Structure is evident on the scale of $0.7^{\circ}$ in RA and $10 \mathrm{~km} \mathrm{~s}^{-1}$ in velocity (halfwidth). Verschuur et al. (1972) found bright cores in HVC $112+2-139$ and HVC $132+23-204$ with dimensions of $0.5^{\circ}$ and velocity widths of $5 \mathrm{~km} \mathrm{~s}^{-1}$. A similar situation was found by Giovanelli et al. (1973) in complexes A, M I, M II, C I and C III. Another characteristic of many of the complexes is the large range of velocities seen in adjacent positions, sometimes as great as $50 \mathrm{~km} \mathrm{~s}^{-1}$. This suggests an intrinsic spread of velocities in the gas constituting the HVC bands. Given a distance, it sets a limit on the lifetime of the bands. For example, taking the main band through $l=140^{\circ}, b=+28^{\circ}$, to be at a distance of $15 \mathrm{kpc}$, a systematic velocity difference of $20 \mathrm{~km} \mathrm{~s}^{-1}$ would separate features by $8^{\circ}$ in $10^{8} \mathrm{yr}-\mathrm{a}$ value consistent with the observations.

The fine structure in the northern HVC bands can be compared with that of the Magellanic stream and the region between the Large and Small Magellanic Clouds. Figure 5, taken from Murray et al. (1974), shows the structure in a cut through the Magellanic stream at $\mathrm{Dec}=-50^{\circ}$. Two main branches of the stream are intersected in this cut - at $R A=00^{\mathrm{h}} 55^{\mathrm{m}},+90 \mathrm{~km} \mathrm{~s}^{-1}$, and $\mathrm{RA}=01^{\mathrm{h}} 23^{\mathrm{m}},+140 \mathrm{~km} \mathrm{~s}^{-1}$. Weaker extended emission surrounds the main peak in each branch.

The average structural parameters of the following types of cloud are compared in Table I: (i) HVCs in the bands, (ii) the Magellanic stream, (iii) the region between the Magellanic clouds and (iv) spiral arm clouds in the galactic plane with $|v| \sim 100 \mathrm{~km} \mathrm{~s}^{-1}$. 


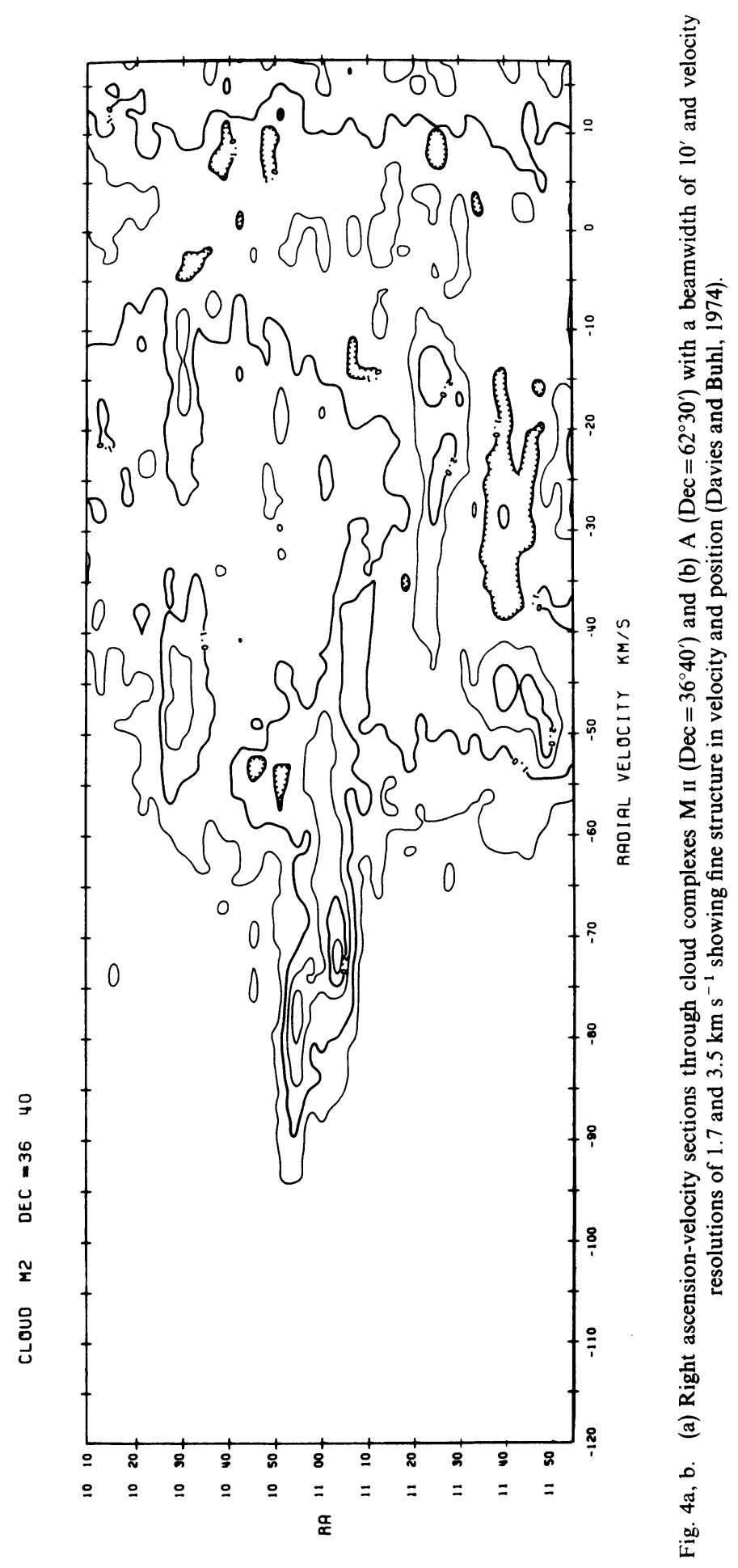




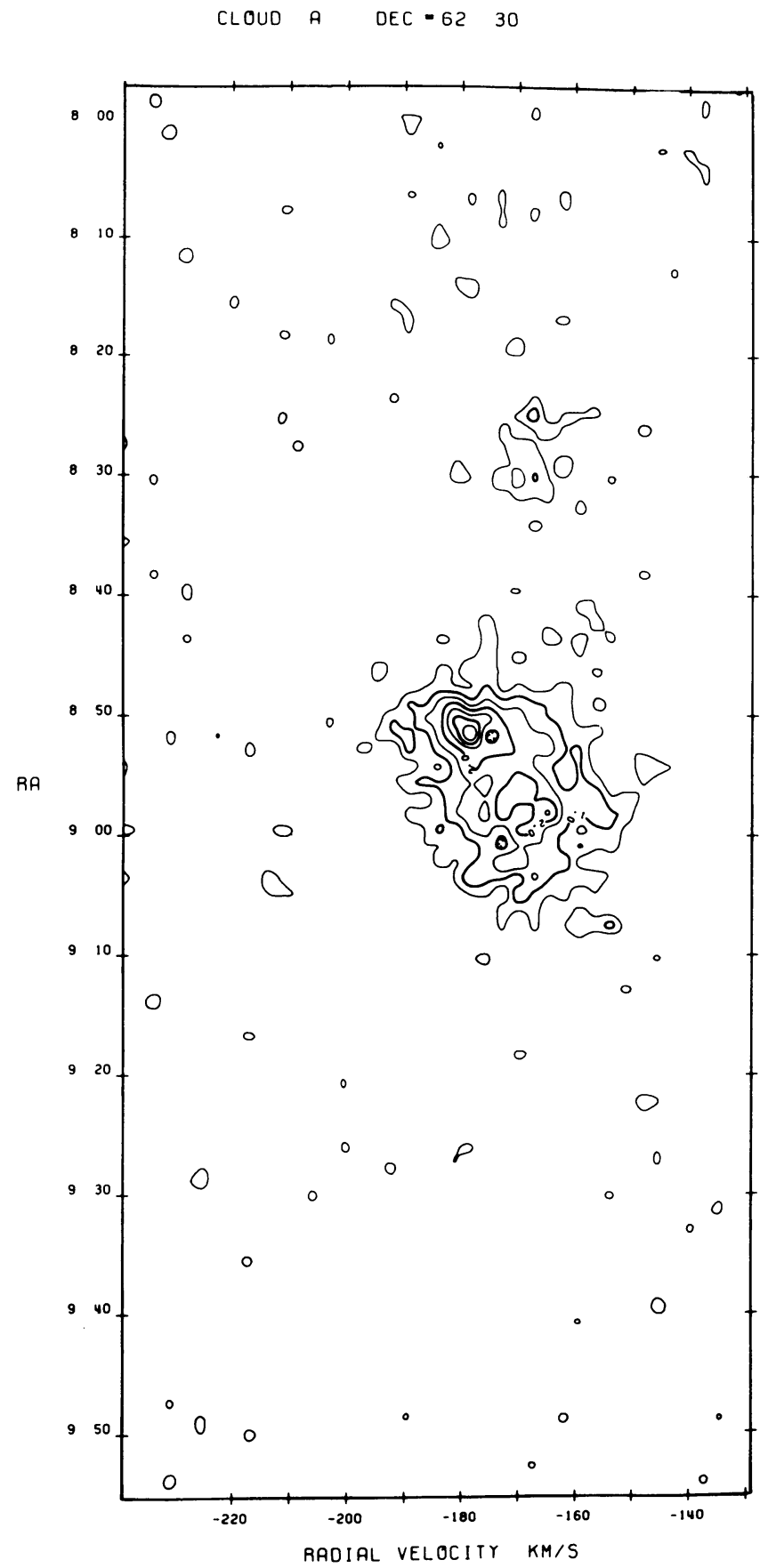

Fig. 4b. 


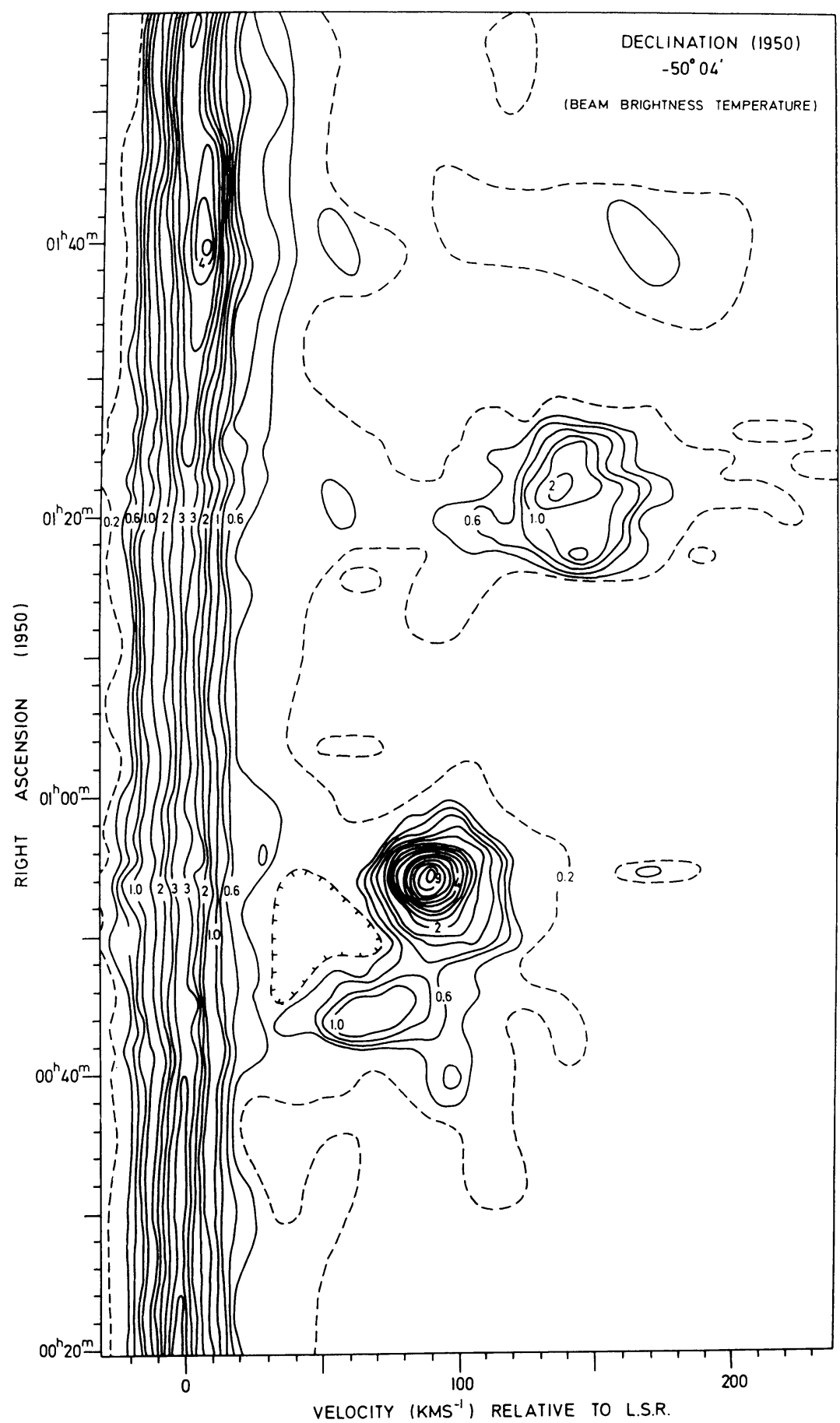

Fig. 5. A right ascension-velocity section through the Magellanic stream at Dec $=-50^{\circ} 04^{\prime}(1950)$ with a resolution of 14 arcmin in angle and $7 \mathrm{~km} \mathrm{~s}^{-1}$ in velocity (Murray et al., 1974). Two branches of the stream are shown in this section, one at $0^{\mathrm{h}} 55^{\mathrm{m}}$ and one at $01^{\mathrm{h}} 23^{\mathrm{m}}$. 
It can be seen that the HVCs have similar structural parameters to the Magellanic streams and the inter-Cloud region. The spiral arm clouds have about half the velocity spread of the other clouds.

A further important consideration is whether the clouds listed in Table I are gravitationally stable. If we take a typical HVC with a dispersion of $8 \mathrm{~km} \mathrm{~s}^{-1}$ (half width $=20 \mathrm{~km} \mathrm{~s}^{-1}$ ) and a diameter of $0.5^{\circ}$ at a distance of $20 \mathrm{kpc}$, the mass required to stabilize the cloud is $\sim 10^{6} M_{\odot}$. However, in a cloud with $N_{\mathrm{H}}=10^{20} \mathrm{~cm}^{-2}$ the total hydrogen mass would be $\sim 2 \times 10^{4} M_{\odot}$ at the assumed distance. We are accordingly left with a deficiency of a factor of $\sim 30$ when account is taken of the contribution due to helium (and possibly an ionized component). This calculation demonstrates, as has been known since the earliest observations, that the HVC complexes are not gravitationally bound at distances of a few tens of kpc. However, the narrow velocity components of the HVCs could well be gravitationally stable with velocity dispersion of $\sim 3 \mathrm{~km} \mathrm{~s}^{-1}$ and a rather greater distance.

TABLE I

Structural parameters of HVCs

\begin{tabular}{lll}
\hline & \multicolumn{1}{l}{ Average half power width } \\
\cline { 2 - 3 } & Velocity $\left(\mathrm{km} \mathrm{s}^{-1}\right)$ & Angle \\
\hline HVCs in bands & $20\left(10^{*}\right)$ & $0.5\left(0^{\circ} 3^{\mathrm{a}}\right)$ \\
$\begin{array}{l}\text { Magellanic stream } \\
\text { Region between Magellanic Clouds }\end{array}$ & 26 & 0.8 \\
$\begin{array}{l}\text { Spiral arm clouds with } \\
|v| \sim 100 \mathrm{~km} \mathrm{~s}^{-1}\end{array}$ & 10 & 0.74 \\
\hline a Velocity structure within a cloud. & & 0.9 \\
\hline
\end{tabular}

Table I also shows that the parameters of the clouds in the Magellanic streams and in the region between the Clouds are similar. Taking a distance of $60 \mathrm{kpc}$ for both, it is again found that the cloud masses are insufficient (by a factor of $\sim 10$ on the average in this case) to make them gravitationally stable.

There is no reason a priori why the HVCs should be gravitationally bound. Indeed, the argument can be inverted to give an age for this clouds in the various complexes. An expansion velocity of $10 \mathrm{~km} \mathrm{~s}^{-1}$ in a cloud complex $2.0^{\circ}$ radius at a distance of $20 \mathrm{kpc}$ leads to an upper limit to the age of $\sim 10^{8} \mathrm{yr}$. Alternatively, the small components within the HVCs with a velocity dispersion of $3 \mathrm{~km} \mathrm{~s}^{-1}$ and a radius of $0.25^{\circ}$ would have a lifetime of $\sim 3 \times 10^{7} \mathrm{yr}$. At the distance of the Magellanic Clouds the ages would be $\sim 3$ times these values. These arguments suggest that we are dealing with clouds produced in the recent past $\left(\sim 10^{8} \mathrm{yr}\right)$ if their distances are $20-60 \mathrm{kpc}$ as the evidence suggests. We shall argue in Section VII that it is not a coincidence that this is of the same order as the time of the estimated perigalacticon passage of the Magellanic Clouds. 


\section{HVCs and Other Phenomena}

Several correlations between HVCs and optical phenomena have been investigated in order to throw light on the nature of HVCs. The observations of interstellar absorption lines in the spectra of high-z $O$ and B stars by Münch and Zirin (1961) showed several intermediate velocity absorbing clouds in the velocity range -70 to $+78 \mathrm{~km} \mathrm{~s}^{-1}$ relative to the LSR. Habing (1969) noted that these intermediate velocity optical components could be found in stars at sufficiently large distances from the galactic plane; the components were also seen in $\mathrm{H}$ I emission spectra taken in the direction of the stars. He concluded that the clouds were at distances of $\sim 1 \mathrm{kpc}$ from the galactic plane. Hulsbosch (1973) reports that Herbig searched unsuccessfully for optical absorption lines in the spectra of several stars lying in the direction of a few HVCs. An intermediate velocity cloud was seen, however, at a distance of between 400 and 1700 pc. All that can be concluded from these studies is that IVCs lie within a distance of $\sim 1 \mathrm{kpc}$; they give no evidence about the distance of HVCs except that they are further than several kpc.

Minkowski et al. (1972) noted that there was a tendency for H II regions of Lynds' catalogue to cluster in the vicinity of high velocity $\left(|v|>100 \mathrm{~km} \mathrm{~s}^{-1}\right)$ gas. They speculate that the excitation may be causally related to the high-velocity gas - a $100 \mathrm{~km} \mathrm{~s}^{-1}$ shock would photoionize substantial amounts of interstellar gas. Until the association is confirmed by velocity measurements of the $\mathrm{H}$ in regions it is too early to pursue this further.

On the theoretical side Silk (1973) has made the proposal that the negative velocity HVCs may be propelled towards the Galaxy by the background soft X-rays. These will ionize the side of the cloud away from the Galaxy (the near side is protected by the Galaxy) and accelerate it inwards by the Oort-Spitzer rocket effect. There are several difficulties with this model: (i) it cannot explain the presence of positive velocity clouds, (ii) it is difficult to obtain a sufficient amount of gas in the neutral state at large distances from the Galaxy in the required X-ray flux and (iii) adequate soft $\mathrm{X}$-rays have not been detected to effectively maintain this process.

\section{Conclusion}

The main observational characteristics of the HVCs have been reviewed above. The salient properties which have to be taken into account in any theories of their origin are: (i) there are extensive areas of both negative and positive velocity gas, (ii) the major part of the HVC gas lies in bands or strings in which there are concentrations having small velocity spread and small angular diameter and (iii) much of the HVC material at low galactic latitudes $\left(|b|<20^{\circ}\right)$ is part of the outer spiral structure of the Galaxy.

One new element in the understanding of the HVCs is the observation by Mathewson et al. of the streams emanating from the Magellanic Clouds. This establishes clearly that the Clouds are being tidally disrupted by the Galaxy during a close approach in which perigalacticon passage must have occurred several times $10^{8} \mathrm{yr}$ 
ago. There is now little doubt about this close approach between the Galaxy and the Magellanic Clouds; in recent years it has been the most widely accepted explanation of the well-known warp in the $\mathrm{H}_{\mathrm{I}}$ distribution in the Galaxy. The timescale and the distance of the perigalacticon passage required for the tidal effects in the Galaxy and the Magellanic Clouds are closely similar. The possible (and likely) contribution of this interaction to material in the vicinity of the Galaxy must be kept in mind in any discussion of HVCs.

More than one origin may have to be sought for the full range of HVC phenomena. Clearly the Magellanic streams have been drawn from the Magellanic Clouds by the tidal influence of the Galaxy. On the other hand, the bulk of the low latitude HVCs are generally agreed to be a part of the galactic spiral structure which also suffers a tidal warp in the outer reaches of the Galaxy. The discussion of the origin of the HVCs centres around the remainder, namely those HVCs at higher latitudes and those at lower latitudes with a large component in their velocities not attributable to galactic rotation. The line of argument favoured by the reviewer would be that this remaining category of HVCs would also be a result of the interaction between the Galaxy and the Magellanic Clouds. Those clouds at higher latitudes in the region above the galactic plane between $l=40^{\circ}$ and $200^{\circ}$ and the positive velocity gas near the plane at $l=250^{\circ}$ to $320^{\circ}$ would be gas originating in the Galaxy and pulled out of the plane by the tidal interaction in the manner discussed by Hunter and Toomre. This would leave the HVCs at the highest latitudes and in the anticentre region which could be either material that essentially has been pulled from the Galaxy or the Magellanic Clouds and is now falling towards the Galaxy. The velocities observed are all energetically possible. It is now necessary to calculate orbits for material drawn out of the Galaxy and the Magellanic Clouds by the encounter and see whether a satisfactory fit can be made to the observed orientation and velocity pattern in this latter group of HVC bands. This is one of the most important tasks to be done to elucidate the origin of thise HVCs.

One is then left to consider the role of the infalling intergalactic gas proposed by Oort. It is one possible mechanism to explain the velocity of those clouds which depart strongly (in the negative sense only) from galactic rotation velocities. The theory also requires an intergalactic medium with a density of $\sim 3 \times 10^{-4} \mathrm{~cm}^{-3}$ and a neutral hydrogen halo surrounding the galactic disk. The existence of an intergalactic medium of this density and a mechanism for ejecting gas from the plane can only be conjectured. Further confirmatory evidence for these phenomena would be of help in assessing the contribution of the intergalactic infall mechanism. One possible check for the existence of infalling intergalactic gas has been suggested by Oort at IAU Symp. 58 where he drew attention to the possible influence of this gas on the dynamics of the Magellanic streams.

\section{References}

Berkhuijsen, E. M., Haslam, C. G. T., and Salter, C. J. : 1971, Astron. Astrophys. 14, 252.

Blaauw, A. and Tolbert, C. R.: 1966, Bull. Astron. Inst. Neth. 18, 405. 
Clutton-Brock, M.: 1972, Astrophys. Space Sci. 17, 292.

Davies, R. D.: 1972, Nature 237, 88.

Davies, R. D.: 1973, Monthly Notices Roy. Astron. Soc. 160, 381.

Davies, R. D. and Buhl, D.: 1974, in preparation.

Dieter, N. H. : 1965, Astron. J. 70, 552.

Dieter, N. H.: 1971, Astron. Astrophys. 12, 59.

Encrenaz, P. J., Penzias, A. A., Gott, R., Wilson, R. W., and Wrixon, G. T.: 1971, Astron. Astrophys. $12,16$.

Eneev, T. M., Kozlov, N. N., and Sunyaev, R. A.: 1973, Astron. Astrophys. 22, 41.

Fejes, I. and Verschuur, G. L.: 1973, Astron. Astrophys. 25, 85.

Habing, H. J.: 1966, Bull. Astron. Inst. Neth. 18, 323.

Habing, H. J.: 1969, Bull. Astron. Inst. Neth. 20, 177.

Hulsbosch, A. N. M.: 1968, Bull. Astron. Inst. Neth. 20, 33.

Hulsbosch, A. N. M.: 1973, Thesis, Sterrewacht te Leiden.

Hulsbosch, A. N. M. and Raimond, E.: 1966, Bull. Astron. Inst. Neth. 18, 413.

Hunter, C. and Toomre, A.: 1969, Astrophys. J. 155, 747.

Kepner, M.: 1970, Astron. Astrophys. 5, 444.

Kerr, F. J.: 1964, Lagonissi Lecture.

Kerr, F. J. and Sullivan, W. T.: 1969, Astrophys. J. 158, 115.

Kerr, F. J. and Westerhout, G. : 1965, Stars and Stellar Systems 5, 167.

Kuilenberg, J. van: 1972a, Astron. Astrophys. 16, 276.

Kuilenberg, J. van: 1972b, Astron. Astrophys. Suppl. 5, 1.

Mathewson, D. S., Clearly, M. N., and Murray, J. D.: 1973, Astrophys. J. 190, 291.

Mathewson, D. S., Meng, S. Y., Brundage, W. D., and Kraus, J. D. : 1966, Astron. J. 71, 863.

Meng, S. Y. and Kraus, J. D.: 1970, Astron. J. 75, 535.

Minkowski, R., Silk, J., and Siluk, R. S.: 1972, Astrophys. J. Letters 175, L1 23.

Muller, C. A., Raimond, E., Schwarz, U. J., and Tolbert, C. R.: 1966, Bull. Astron. Inst. Neth. Suppl. 1, 213.

Münch, G. and Zirin, H.: 1961, Astrophys. J. 133, 11.

Murray, J. D., Davies, R. D., Mathewson, D. S., and Cleary, M. N.: 1974, in preparation.

Oort, J. H.: 1966, Bull. Astron. Inst. Neth. 18, 421.

Oort, J. H. : 1969, Nature 224, 1158.

Oort, J. H.: 1970, Astron. Astrophys. 7, 381.

Rickard, J. J.: 1968, Astrophys. J. 152, 1019.

Savedoff, M. P., Hovenier, J. W., and van Leer, B. : 1967, Bull. Astron. Inst. Neth. 19, 107.

Silk, J.: 1973, Astron. Astrophys., in press.

Tolbert, C. R.: 1971, Astron. Astrophys. Suppl. 3, 349.

Toomre, A. and Toomre, J. : 1972, Astrophys. J. 178, 623.

Verschuur, G. L. : 1969, Astrophys. J. 156, 771.

Verschuur, G. L.: 1973, Astron. Astrophys. 22, 139.

Verschuur, G. L., Cram, T. R., and Giovanelli, R.: 1972, Astrophys. Letters 11, 57.

Wannier, P. and Wrixon, G. T.: 1972, Astrophys. J. Letters 173, L119.

Wannier, P., Wrixon, G. T., and Wilson, R. W.: 1972, Astron. Astrophys. 18, 224.

Weaver, H.: 1971, in W. Becker and G. Contopoulos (eds.), 'The Spiral Structure of Our Galaxy', IAU Symp. 38, 126.

Weaver, H. F. and Williams, D. R. W.: 1973, Astron. Astrophys. Suppl. 8, 1.

Wright, A. E.: 1972, Monthly Notices Roy. Astron. Soc. 157, 309.

R. D. Davies

University of Manchester,

Nuffield Radio Astronomy Laboratories, Jodrell Bank, Cheshire SK11 9DL, United Kingdom

(Discussion follows the paper by D. S. Mathewson et al., p. 623.) 